\title{
Neuralgia occipital: relato de caso e revisão
}

\section{Occipital Neuralgia: Case Report and Review}

\author{
Marcelo José da Silva de Magalhães ${ }^{1}$ Maeds Soares Cardoso ${ }^{2}$ Isabelle Lopes Gontijo ${ }^{2}$ \\ Tainá Fernandes Darce ${ }^{2}$
}
${ }^{1}$ Mestrando em Neurofisiologia pela Inforium; Professor de Medicina das Faculdades Unidas do Norte de Minas (FUNORTE) e Faculdades Integradas Pitágoras Montes Claros (Fip-Moc); Neurocirurgião Geral e Cirurgião de Nervos Periféricos do Hospital Aroldo Tourinho,
Montes Claros, MG, Brasil
2 Estudante de Medicina, FUNORTE, Montes Claros, MG, Brasil

\begin{abstract}
Address for correspondence Marcelo José da Silva de Magalhães, MD, Rua Reginaldo Ribeiro ${ }^{\circ}{ }^{\circ} 69$, Centro, Montes Claros, MG, Brasil CEP: 39400113 (e-mail: marcelo7779@yahoo.com.br).
\end{abstract}

Arq Bras Neurocir 2016;35:101-104.

\section{Resumo}

\section{Palavras-Chave}

- occipital neuralgia

- nervo occipital maior

- cefaleia
Neuralgia occipital (NO) é uma causa incomum de cefaleia caracterizada por dor paroxística, do tipo pontada, que se irradia para a região occipital. O objetivo deste artigo é relatar o caso de uma paciente com NO e descrever a técnica cirúrgica utilizada. O estudo compreende um levantamento bibliográfico para o conhecimento e melhor abordagem sobre o assunto. Com base na literatura, observa-se que a etiologia pode variar desde traumas, infecções, cirurgias de base de crânio, compressões de nervos ou vértebras até alterações degenerativas e anomalias congênitas. Porém, em sua maioria, os casos são idiopáticos. Apesar de o diagnóstico ser essencialmente clínico, é fundamental que sejam excluídos outros tipos de cefaleias primárias. De acordo com a gravidade e o tempo de evolução do caso, o tratamento da NO pode basear-se em bloqueios nervosos, medicamentos - como anti-inflamatórios não-esteroides e relaxantes musculares - ou cirurgias. Entre os procedimentos cirúrgicos disponíveis, encontram-se a descompressão do nervo occipital maior, ablação por radiofrequência e implantação de neuroestimulador.

Occipital Neuralgia (ON) is an uncommon cause of headache, characterized by paroxysmal pain, stabbing that radiates to occipital region. This article aims at reviewing the literature to the approach to the subject and performs the case report of patient who present with ON and underwent a surgical treatment. Based on the literature and analysis showed the etiology may vary from trauma, infections, skull base surgery, compression of nerves or vertebrae to degenerative changes and congenital anomalies. However, most cases are idiopathic. Although the diagnosis is essentially clinical, it is essential that other types of primary headaches are excluded. According to severity and the time course of the case, the treatment of ON may be based on nerve blocks, medications like non-steroidal anti-inflammatory drugs and muscle relaxants. Surgical treatment for $\mathrm{ON}$ are nerve decompression, pulsed radiofrequency ablation and stimulator implantation. received

March 3, 2015

accepted

August 28, 2015

published online

February 17, 2016
DOI http://dx.doi.org/

10.1055/s-0035-1571173. ISSN 0103-5355.
Copyright $@ 2016$ by Thieme Publicações License terms

Ltda, Rio de Janeiro, Brazil 


\section{Introdução}

Segundo o International Headache Society, a neuralgia occipital (NO) é definida como uma dor paroxística, do tipo pontada, que se irradia para a região occipital. ${ }^{1}$ Essa patologia, primeiramente descrita em 1821 por Beruto et al., é considerada causa incomum de cefaleia. ${ }^{2}$

Embora em sua maioria os casos de NO sejam idiopáticos, traumas, infecções, cirurgias de base de crânio, compressões de nervos, compressões vertebrais, alterações degenerativas e anomalias congênitas podem ser fatores causais dessa enfermidade. $^{2}$

Deve-se ressaltar que apesar de o diagnóstico da NO ser essencialmente clínico, ele é muitas vezes dificultado devido à variedade de sinais e sintomas que podem acometer o paciente. ${ }^{3}$ Isso corrobora o fato de esta nevralgia poder ser confundida com outros tipos de cefaleias primárias. $^{3}$

Entre as opções terapêuticas para a NO, bloqueios nervosos ou cirurgias do nervo occipital são considerados tratamentos de primeira escolha. ${ }^{3}$ Medicamentos - como anti-inflamatórios não esteroides, anticonvulsivantes e relaxantes musculares -, descompressão e ablação por radiofrequência pulsada também podem ser utilizados. ${ }^{3}$

O objetivo deste trabalho é apresentar o relato de um caso sobre o tema, além de fazer revisão deste na literatura médica, dando ênfase às opções de tratamento.

O paciente em questão concordou em ceder seus dados e fotos do procedimento cirúrgico, assinando o consentimento livre e esclarecido. A realização do trabalho não acarretou dano de qualquer natureza para o paciente. Nenhum dos autores do trabalho apresenta conflito de interesse.

\section{Relato de Caso}

Paciente de 38 anos, sexo feminino, casada, com relato de cefaleia occipital direita com 4 anos de duração. Cefaleia em caráter de aperto, constante, de forte intensidade e diária. Mesmo em uso de inúmeros medicamentos, mantinha o quadro álgico. Seguia o tratamento com: Lyrica ${ }^{\circledR}, 150 \mathrm{mg}$ de 8/8 h; Cymbalta ${ }^{\circledR}, 60$ mg de 12/12h; Rivotril ${ }^{\circledR}, 2$ mg de 8/ $8 \mathrm{~h}$; Lisador ${ }^{\circledR}$, de $8 / 8 \mathrm{~h}$; Vimovo ${ }^{\circledR} 500,20 \mathrm{mg}$; e Tylex ${ }^{\circledR}$, $60 \mathrm{mg}$ de $8 / 8 \mathrm{~h}$. A paciente é portadora de intolerância a glicose, obesidade e transtorno depressivo maior. Foi afastada de seu trabalho há cerca de 2 anos devido ao quadro de cefaleia.

Exame neurológico não mostrava alterações focais, exceto pela presença de sinal de Tinel em região do nervo occipital maior à direita, o qual reproduzia o padrão de dor que a paciente vinha apresentando.

Após a suspeita do diagnóstico de neuralgia occipital, a paciente foi submetida a bloqueio anestésico na região do nervo occipital maior à direita, com alívio imediato dos sintomas, o que corroborou o diagnóstico.

A ressonância magnética de crânio afastou causas secundárias de cefaleia. Revisão laboratorial revelou velocidade de hemossedimentação de $27 \mathrm{~mm}$ após 1 hora. Foi orientada sobre as possibilidades de tratamento para a neuralgia do nervo occipital. Optou-se pela rizotomia a céu aberto.

A paciente foi submetida à anestesia geral e colocada em decúbito ventral. A incisão foi realizada em "U" invertido. Por meio de dissecção cuidadosa dos planos, foi identificado o nervo occipital maior, a cerca de $6 \mathrm{~cm}$ da protuberância occipital externa (-Fig. 1). Não foi encontrado neuroma nem alterações teciduais em trajeto do nervo na região occipital. A cirurgia foi realizada com sucesso e obteve melhora imediata dos sintomas.

Em consulta de retorno, 9 meses após a cirurgia, a paciente mantinha controle dos sintomas e não apresentava complicações decorrentes do procedimento cirúrgico.

\section{Discussão}

A NO se refere à dor em território occipital ou cervical causada pela estimulação e/ou compressão do nervo occipital maior. ${ }^{4}$ A dor tem como características ser unilateral e acometer a região occipital que apresenta inervação pelo nervo occipital maior. ${ }^{5}$ Essa enfermidade pode ser relatada pelo paciente como dor em padrão de queimação ou em pontadas, podendo, inclusive, estar associada à sensação de "choque", parestesia, fotofobia, náuseas e vômitos. Tem início após posição sustentada da cabeça por longo período de tempo, como ocorre em leituras prolongadas ou posições inadequadas durante o sono. A duração da dor pode variar de horas a dias. ${ }^{7}$ Apresenta discreta predominância no sexo feminino. ${ }^{7}$

Fatores etiológicos traumáticos, degenerativos, oncológicos ou idiopáticos correspondem, respectivamente, a 72, 14 e $14 \%$ dos casos. ${ }^{8}$ Artrite cervical, compressão nervosa, traumatismo raquimedular e malformações de Chiari I são alguns dos diagnósticos diferenciais dessa patologia. ${ }^{5,7}$

O conhecimento neuroanatômico dos nervos occipital maior e menor possibilitou a investigação etiológica e a melhoria do arsenal terapêutico da NO.,7 0 nervo occipital maior origina-se de ramos posteriores dos segmentos do nervo espinhal C2 após sua emergência do gânglio sensitivo. Adquire um trajeto recorrente em direção à borda inferior do músculo oblíquo inferior da cabeça , contornando-o, e trafega em direção superior, obtendo neste momento íntima relação com o músculo semiespinal da cabeça. A partir deste ponto, cruza o referido músculo, mantendo o seu trajeto ascendente e estabelecendo nova relação ao passar sob o músculo trapézio. Em sua última porção, o nervo atravessa as fibras do trapézio, exteriorizando-se no tecido celular subcutâneo. ${ }^{1,2,7}$

O diagnóstico da neuralgia occipital é alcançado principalmente pelo exame clínico; nenhum exame de imagem é comprovadamente bem-sucedido no diagnóstico. ${ }^{9}$ Os critérios para NO, segundo o International Headache Society, são:

1. dor referida na distribuição dos nervos occipitais maior ou menor;

2. dor em pontadas, podendo ou não persistir sensação dolorosa entre os paroxismos;

3. sensação dolorosa à palpação sobre o nervo afetado; 


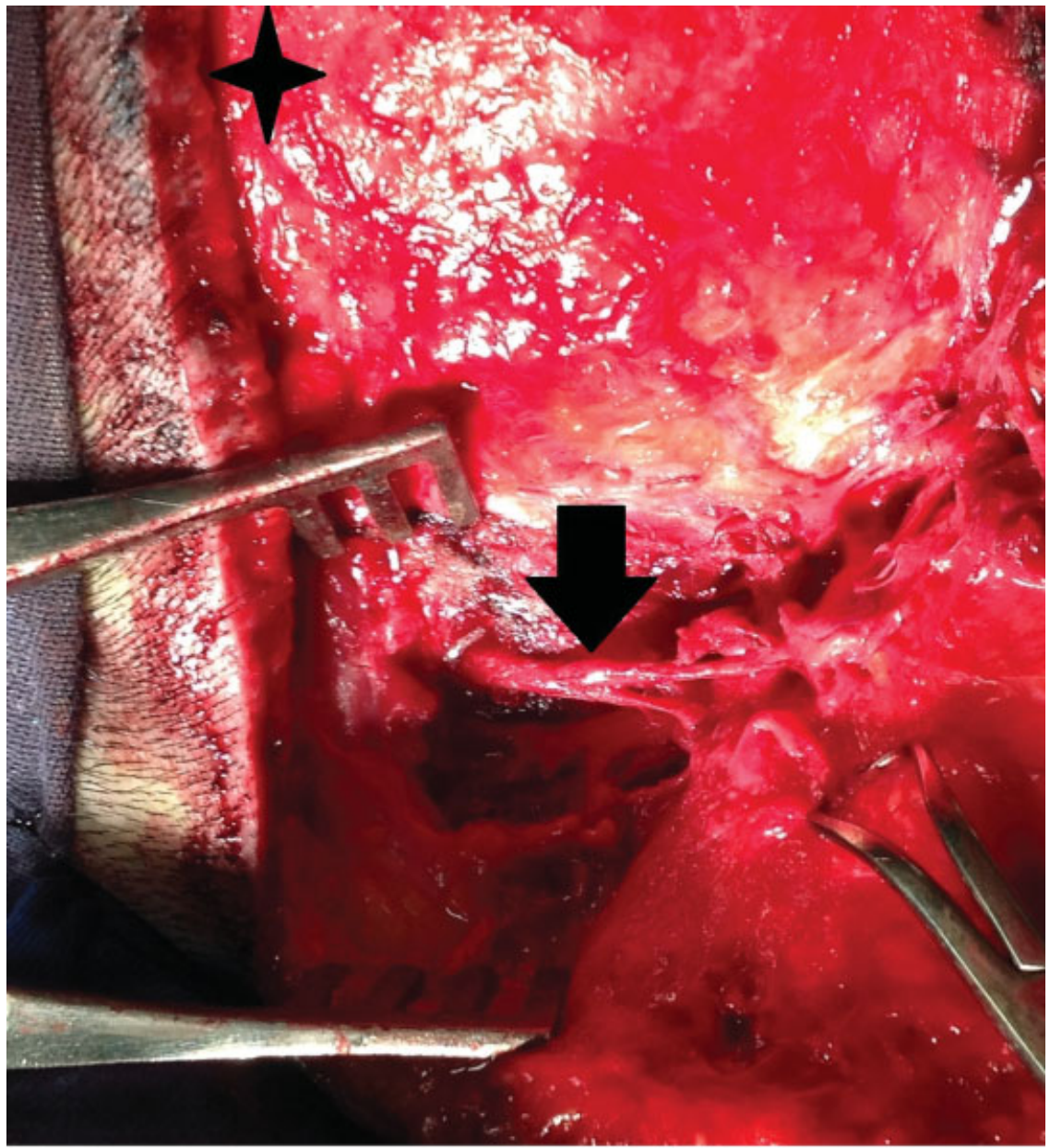

Fig. 1 Fotografia intraoperatória da região occipital direita. A seta indica o nervo occipital maior, e a estrela, a protuberância occipital externa.

4. dor aliviada temporariamente pelo bloqueio anestésico local do nervo apropriado. ${ }^{7}$

Possíveis zonas de irritação ou encarceramento do nervo occipital maior incluem:

1. local onde o nervo occipital maior emerge do ramo dorsal de $\mathrm{C} 2$ entre o atlas e o áxis;

2. trajeto onde o nervo cursa entre os músculos oblíquo inferior da cabeça e semiespinal da cabeça;

3. local onde o nervo perfura o corpo do músculo semiespinal da cabeça;

4. local de saída do nervo no nível do músculo trapézio. ${ }^{9}$

Foram estabelecidas diferentes abordagens terapêuticas para a NO, como tratamento conservador com colar cervical, analgésicos, bloqueio percutâneo do nervo, neuroestimulação, e ablação por radiofrequência. ${ }^{2} \mathrm{~A}$ infiltração do nervo occipital maior, utilizada como diagnóstico e tratamento da dor, pode ser realizada com bupivacaína $0,5 \%$, associada ou não a metilprednisolona, em um ponto localizado a $3 \mathrm{~cm}$ lateralmente e $2 \mathrm{~cm}$ inferiormente à protuberância occipital externa. ${ }^{2,7}$ Esse bloqueio do nervo mostrou resultados positivos no tratamento de outros tipos de cefaleia. Uma hipótese que justifica esta melhora é a proximidade dos neurônios sensoriais da espinha cervical superior com neurônios do núcleo caudado trigeminal, e a convergência do sensório para neurônios do núcleo causado trigeminal de ambas as fibras cervical e trigeminal. ${ }^{9}$ Há poucas complicações com o bloqueio percutâneo do nervo pela sua localização superficial. ${ }^{1}$ Entretanto, é possível a injeção intravascular. ${ }^{1}$

Em um estudo utilizando 92 pacientes tratados com infiltração percutânea de lidocaína e esteroide, foi observada melhora em $87 \%$ dos pacientes, com recorrência em 31,5\% deles. Entre aqueles que apresentaram melhora inicial, a dor geralmente persistiu por um prazo de 1 a 8 meses. $^{2}$

A neuroestimulação do nervo occipital tem sido relatada como uma modalidade não ablativa. ${ }^{8}$ Um intensificador de imagem pode ser utilizado para a inserção dos eletrodos estimuladores. A neuroestimulação tem eficácia no controle da dor, variando de 60 a $90 \%{ }^{8}$ Entretanto, pode haver mal posicionamento dos eletrodos, por ser técnica cega, ou migração dos mesmos, em 10 a 70\% dos casos, com subsequente perda da estimulação e frequente indicação de revisão cirúrgica. ${ }^{8}$ Um estudo utilizando uma técnica aberta, apesar da pequena amostra, demonstrou ser uma 
104 Neuralgia occipital: relato de caso e revisão Magalhães et al.

modalidade segura, simples e efetiva em manter contato direto entre o eletrodo e o tronco do nervo, em um paciente previamente anestesiado. $^{8}$

Outro estudo, realizado por Blume et al, utilizou a técnica de ablação por radiofrequência. ${ }^{2}$ Após 6 a 24 meses de seguimento, demonstrou-se melhora dos sintomas em $90,7 \%$ dos pacientes sem anormalidades da medula espinal e em 74,4\% daqueles com anormalidades. ${ }^{2}$

Apesar da pequena amostra, um estudo avaliou retrospectivamente 17 pacientes submetidos a rizotomia a céu aberto. Não foram observadas maiores complicações, mas incluem-se como potenciais riscos: infecção, paralisia, fístula liquórica e paresia do músculo trapézio pela lesão do nervo espinhal acessório. $^{2}$

A utilização da toxina botulínica tem-se mostrado eficaz como terapia da NO quando associada ao traumatismo raquimedular. ${ }^{6}$ Vale ressaltar que seu uso já é consagrado no tratamento de outros tipos de cefaleia, como a enxaqueca. ${ }^{6}$

\section{Conclusão}

A neuralgia occipital requer uma cuidadosa investigação para o estabelecimento de seu diagnóstico. Embora existam vários métodos de tratamento, a rizotomia a céu aberto pode ser uma opção para esta patologia, tendo a vantagem de ser pouco dispendiosa. Sua técnica exige conhecimento anatômico para o devido reconhecimento das estruturas anatô- micas envolvidas. Estudos para melhor definição dos resultados funcionais a longo prazo ainda são necessários.

\section{Referências}

1 Ward JB. Greater occipital nerve block. Semin Neurol 2003;23(1): 59-62

2 Kapoor V, Rothfus WE, Grahovac SZ, Amin Kassam SZ, Horowitz MB. Refractory occipital neuralgia: preoperative assessment with CT-guided nerve block prior to dorsal cervical rhizotomy. AJNR Am J Neuroradiol 2003;24(10):2105-2110

3 Benavides N. Occipital Neuralgia: A Literature Review of Current Treatments from Traditional Medicine to CAM Treatments. Chesterfield: Logan University; 2011

4 Pan C, Tan G. Forty-two cases of greater occipital neuralgia treated by acupuncture plus acupoint-injection. J Tradit Chin Med 2008; 28(3):175-177

5 Kemp WJ III, Tubbs RS, Cohen-Gadol AA. The innervation of the scalp: A comprehensive review including anatomy, pathology, and neurosurgical correlates. Surg Neurol Int 2011;2:178

6 Barna S, Hashi M. Occipital Neuralgia. Pain Management Rounds 2004;1(7):1-5

7 Piovesan EJ, Werneck LC, Kowacs PA, et al. Nevralgia do occipital maior associada a lesão osteolítica occipital. Relato de caso. Arq Neuropsiquiatr 1999;57(1):114-119

8 Magown P, Garcia R, Beauprie I, Mendez IM. Occipital nerve stimulation for intractable occipital neuralgia: an open surgical technique. Clin Neurosurg 2009;56:119-124

9 Cho JCS, Haun DW, Kettner NW. Sonographic evaluation of the greater occipital nerve in unilateral occipital neuralgia. J Ultrasound Med 2012;31(1):37-42 